\title{
Plasma proatrial natriuretic factor (1-98) concentration after myocardial infarction: relation to indices of cardiac and renal function
}

\author{
Vernon V S Bonarjee, Torbjørn Omland, Dennis W T Nilsen, Kenneth Caidahl, \\ Johan A Sundsfjord, Kenneth Dickstein
}

\begin{abstract}
Objectives-(a) To assess the relation between plasma concentrations of proatrial natriuretic factor (1-98) and non-invasively derived indices of left ventricular systolic and diastolic performance and (b) to assess the potential confounding effect of renal function and age on this relation in patients with acute myocardial infarction.
\end{abstract}

Design-Cross sectional comparison of biochemical and echocardiographic indices of cardiac function.

Setting-Norwegian central hospital.

Patients-Sixty four patients with acute myocardial infarction.

Main outcome measures-Relation between plasma proatrial natriuretic factor (1-98) concentrations and echocardiographic indices of left ventricular systolic function as assessed by univariate and multivariate linear regression analysis. Sensitivity and specificity of plasma proatrial natriuretic factor (1-98) concentration as a measure of left ventricular systolic and diastolic dysfunction.

Results-Plasma proatrial natriuretic factor (1-98) concentrations were significantly related to left ventricular ejection fraction $(r=-0.33 ; P=0.008)$, age $(r=$ $0.43 ; P<0.001)$, and creatinine clearance $(r=-0.53 ; P<0.001)$. In a multivariate model left ventricular ejection fraction and creatinine clearance were both independently related to plasma values. The mean concentration of proatrial natriuretic factor (1-98) was significantly higher in patients with an ejection fraction of $<40 \%$ than in those with an ejection fraction of $\geqslant 40 \%$ (1876 (1151) $v 1174$ (530) $\mathrm{pmol} / \mathrm{l} ; P=0.03$ ) and in patients with an abnormal transmitral $E / A$ ratio ( $<0.65$ or $>1.65$, where $E / A$ is ratio of peak early filling velocity to peak atrial component) compared with those with a normal ratio (1572 (895) $v 1137$ (523) pmol/1, respectively; $P=0.02$ ). When patients were subdivided according to the median concentration of proatrial natriuretic factor $(1192 \mathrm{pmol} / \mathrm{l})$ the sensitivity and specificity were $89 \%$ and $56 \%$ respectively for detecting a left ventricular ejection fraction of $<40 \%$ and $75 \%$ and $61 \%$ respectively for detecting an abnormal E/A ratio. Concentrations below the median had a negative predictive value of $97 \%$ in excluding an ejection fraction of
$<40 \%$ and of $84 \%$ in excluding an abnormal E/A ratio.

Conclusion-These results suggest that soon after myocardial infarction left ventricular ejection fraction and indices of renal function are independently related to plasma concentrations of proatrial natriuretic factor (1-98). Plasma concentrations of proatrial natriuretic factor (1-98) seem to reflect renal and cardiac performance rather than specific haemodynamic variables assessed by noninvasive methods. Plasma proatrial natriuretic factor (1-98) measurements may be a useful screening tool to identify patients with normal cardiac function soon after myocardial infarction.

(Br Heart f 1995;73:511-516)

Keywords: proatrial natriuretic factor (1-98); myocardial infarction; cardiac function; renal function.

Left ventricular end systolic and end diastolic volumes as well as ejection fraction, a commonly used index of cardiac systolic performance, are major determinants of survival after acute myocardial infarction. ${ }^{1-3}$ Data from recent clinical trials have shown that early intervention with converting enzyme inhibitors may reduce left ventricular dilatation and mortality in patients with left ventricular systolic impairment ${ }^{45}$ or symptomatic heart failure ${ }^{6}$ after acute myocardial infarction, as well as in patients with chronic symptomatic and asymptomatic left ventricular dysfunction. ${ }^{7-9}$ Early intervention with intravenous enalaprilat followed by oral enalapril failed to reduce mortality at six months in an unselected group of patients with acute myocardial infarction, ${ }^{10}$ despite attenuation of left ventricular dilatation. ${ }^{11}$ Oral captopril and lisinopril treatment initiated within 24 hours after a myocardial infarction resulted in a small but significant reduction in mortality at 5-6 weeks in two recently completed trials. ${ }^{12} 13$

As the beneficial effect of early treatment with converting enzyme inhibitors is more pronounced in a subgroup of patients with left ventricular dysfunction, a major objective in the care of patients surviving the acute phase of myocardial infarction should be to identify those who are most likely to benefit from such treatment. Moreover, long term treatment may be indicated in patients with asymptomatic left ventricular dysfunction. Although echocardiographic or radionuclide investigations 
are important tools in assessing left ventricular performance, the costs and skills required may limit their usefulness. Evaluation of other methods for assessing left ventricular function may therefore have important practical implications.

Atrial natriuretic factor is released in response to atrial stretch, and its plasma concentrations are raised in patients with left ventricular dysfunction. ${ }^{14}$ Recent data suggest that circulating proatrial natriuretic factor (1-98), the $\mathrm{N}$ terminal fragment of the atrial natriuretic factor prohormone (126 amino acids) may be a more sensitive and specific marker of asymptomatic left ventricular dysfunction than the biologically active $\mathrm{C}$ terminal fragment (atrial natriuretic factor). ${ }^{15}$ Furthermore, owing to its greater stability and subsequent higher plasma concentrations, which permits a simplified analytic procedure, ${ }^{1617}$ proatrial natriuretic factor (1-98) may be a more versatile indicator of left ventricular function in clinical practice than atrial natriuretic factor.

Plasma concentrations of atrial natriuretic peptides are dependent on cardiac performance. Before proatrial natriuretic factor (1-98) can be adopted as an index of left ventricular function routinely, its relation to various indices of cardiac performance and other potentially confounding factors should be established. Consequently, the main objectives of our study were to assess the relation between proatrial natriuretic factor measurements and indices of left ventricular systolic function and to consider the possible confounding effects of age and renal function. Furthermore, we wanted to assess the sensitivity and specificity of proatrial natriuretic factor (1-98) as a means of identifying patients with systolic dysfunction defined as a left ventricular ejection fraction of $<40 \%$ and diastolic dysfunction defined as $\mathrm{E} / \mathrm{A}$ ratio $<0.65$ or $\mathrm{E} / \mathrm{A}$ ratio $>1 \cdot 65 . .^{18} 19$

\section{Patients and methods}

STUDY DESIGN

Patients with a documented myocardial infarction admitted to the coronary care unit over a recruitment period of six months were included in this study. Venous blood for determination of plasma proatrial natriuretic factor (1-98) concentration was obtained on day 3 after the onset of symptoms and echocardiography was performed between day 2 and day 5 after the start of symptoms. All patients participated in the cooperative new Scandinavian enalapril survival study (CONSENSUS II), a randomised, placebo controlled, double blind study investigating the effect on mortality at six months of enalapril treatment initiated soon after myocardial infarction. ${ }^{10}$ The study was approved by the regional ethics committee, and informed consent was obtained from all participating patients.

Inclusion criteria to the study were chest pain of more than 20 minutes duration in combination with acute myocardial infarction shown on standard electrocardiography or enzymatic evidence of myocardial necrosis, or both. Major exclusion criteria were a supine blood pressure below 105/65 mm Hg, cardiogenic shock, a need for pressor support, haemodynamically significant valvar disease, and severe congestive heart failure (New York Heart Association class IV).

\section{BLOOD SAMPLING PROCEDURES AND}

\section{HORMONAL ASSAYS}

Blood samples were drawn by direct venepuncture after at least 30 minutes of supine rest and collected into chilled tubes containing ethylenediaminetetraacetic acid (EDTA) and aprotinin (500 kallikrein inactivator units $/ \mathrm{ml}$ blood). The test tubes were centrifuged and plasma samples were stored at $-70^{\circ} \mathrm{C}$ until analysed for proatrial natriuretic factor (1-98). This analysis was performed with a specific radioimmunoassay without previous extraction. ${ }^{17}$ Creatinine clearance was calculated based on the patient's weight, age, and serum creatinine concentration on admission to hospital. ${ }^{20}$

\section{ECHOCARDIOGRAPHIC EXAMINATIONS}

Echocardiography was performed between day 2 and day 5 after myocardial infarction using a Vingmed CFM 750 (Vingmed Sound, Horten, Norway) machine. Patients were examined in the left lateral supine position by experienced physicians according to the recommendations of the American Society of Echocardiography. ${ }^{21}$ Cross sectional apical two and four chamber views were used for volume measurements. Mitral flow velocities were calculated from pulsed wave Doppler signals recorded at the tip of the mitral valves in the four chamber view. All recordings were stored on video tape from which complete cardiac cycles of cross sectional images as well as mitral Doppler signals were digitised for computerised analysis. Area tracings of two and four chamber images were performed by a single observer, and biplane systolic and diastolic volumes were calculated from these area measurements by the disc summation method (modified Simpson's rule). Ejection fractions (calculated as (diastolic volume systolic volume)/(diastolic volume)) were derived from these echocardiographic volumes. The method and the variation of measurements have been reported in detail previously. ${ }^{11}$ The peak early filling velocity (E wave) and peak atrial component (A wave) of the mitral Doppler signals were measured by manual tracings of computerised signal images performed by a single observer. The E/A ratio was calculated from these measurements.

\section{STATISTICS}

Results are presented as means (SD). As the plasma proatrial natriuretic factor (1-98) concentrations were not normally distributed, values were $\log (\ln )$ transformed. The relation between proatrial natriuretic factor (1-98) and left ventricular ejection fraction were examined by linear regression analysis. Both 
forward and backward procedures were performed in multivariate models.

The sensitivity of a defined plasma proatrial natriuretic factor (1-98) concentration in detecting left ventricular systolic dysfunction (ejection fraction $<40 \%$ ) or diastolic dysfunction (defined as E/A ratio $<0.65$ or $>1.65$ ), or both, was calculated as the proportion of patients with dysfunction who had a plasma proatrial natriuretic factor (1-98) concentration above the defined value. Specificity was similarly calculated as the proportion of patients without dysfunction who had a plasma proatrial natriuretic factor (1-98) concentration below the defined value. The positive predictive value of a defined plasma proatrial natriuretic factor (1-98) concentration to predict systolic and diastolic dysfunction as defined above was calculated as the proportion of patients with a plasma proatrial natriuretic factor (1-98) value above the selected concentration who had dysfunction. The negative predictive value was similarly calculated as the proportion of patients with a plasma proatrial natriuretic factor (1-98) below the selected concentration who did not have dysfunction.

\section{Results}

Complete echocardiographic and biochemical data were available from 64 patients ( 47 men and 17 women) whose average age was 67 (9). Nineteen patients (30\%) had a history of a previous myocardial infarction, 26 patients (41\%) had a history of angina pectoris, two patients $(3 \%)$ had a history of chronic congestive heart failure, 12 patients (19\%) were receiving treatment for hypertension at the time of admission, and five patients ( $8 \%$ ) received treatment for diabetes mellitus.

New pathological $Q$ waves were observed in 39 patients. According to electrocardiographic criteria anterior infarction (including lateral) was diagnosed in 33 patients and non-anterior (inferior, posterior, or undefined) in 31 .

Medical treatment during primary admission to hospital included thrombolysis with streptokinase in 36 patients, intravenous mor-

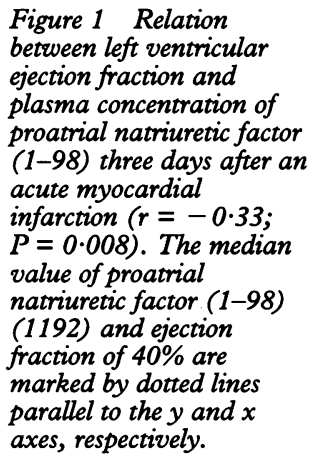

Figure 1 Relation between left ventricular ejection fraction and plasma concentration of proatrial natriuretic factor (1-98) three days after an acute myocardial infarction $(r=-0 \cdot 33$; $P=0.008)$. The median value of proatrial natriuretic factor (1-98) (1192) and ejection fraction of $40 \%$ are marked by dotted lines parallel to the $y$ and $x$ axes, respectively.

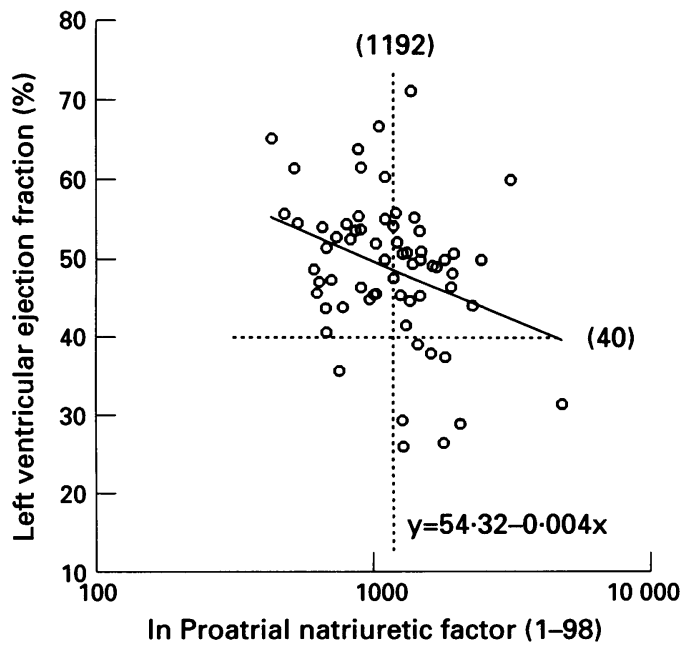

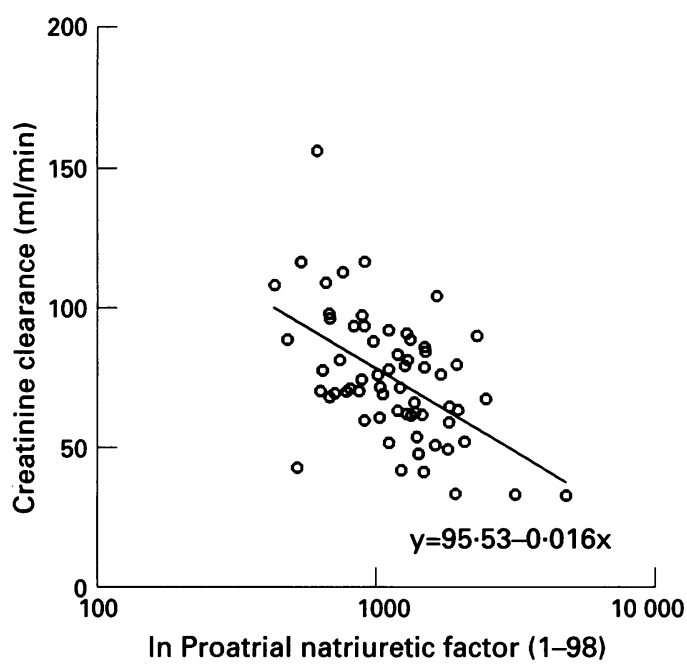

Figure 2 Relation between creatinine clearance and plasma concentration of proatrial natriuretic factor (1-98) three days after an acute myocardial infarction $(r=-0.53 ; P=<0.001)$.

phine in $54, \beta$ blockers in 36 , the angiotensin converting enzyme inhibitor enalapril in 32 , and frusemide in 30 . Sixteen patients developed pulmonary congestion that was treated with diuretics during their stay in hospital, but only one patient developed overt pulmonary oedema.

Left ventricular ejection fraction averaged $49 \%(9 \%)$ (range $26-71 \%$ ) and the transmitral E/A ratio $0.98(0.48)$ (range $0.39-3.57)$. The mean peak serum creatine kinase $M B$ activity was 136 (119) IU/l, the mean plasma proatrial natriuretic factor (1-98) concentration on day 3 after the onset of symptoms 1273 (685) pmol/1, and mean creatinine clearance $74(23) \mathrm{ml} / \mathrm{min}$.

In univariate analyses age $(r=0.43$; $P<0.001)$, left ventricular ejection fraction $(r=-0.33 ; \mathbf{P}=0.008)$, and creatinine clearance $(r=-0.53 ; \mathrm{P}<0.001)$ were all significantly related to plasma proatrial natriuretic factor (1-98) concentration. These relations, except that of age, are shown in figures 1 and 2 , respectively. When introduced into a multivariate regression model left ventricular ejection fraction and creatinine clearance remained significantly and independently related to plasma proatrial natriuretic factor (1-98) concentrations (table 1). Age was not included in the final model. According to the model, $37 \%$ of the variability of plasma proatrial natriuretic factor (1-98) concentrations could be accounted for by the variability in left ventricular ejection fraction and creatinine clearance $\left(R^{2}=0.37\right)$. When creatinine clearance was omitted from the model, age was significantly related to plasma proatrial natriuretic factor (1-98) concentrations independently of left ventricular ejection fraction. Age, however, correlated inversely with creatinine clearance $(r=-0.63 ; \mathrm{P}<0.001)$ and positively with proatrial natriuretic factor (1-98) $(r=0.43 ; P<0.001)$, suggesting that age was a confounder of the relation between proatrial natriuretic factor (1-98) and renal function.

In a multivariate analysis with left ventricular ejection fraction as the dependent variable 
Table 1 Multivariate relation between enplasma concentrations of proatrial natriuretic factor (1-98) concentrations and potential predictor variables

\begin{tabular}{llll}
\hline Variable & Coefficient & $S E$ & Pvalue \\
\hline Left ventricular ejection fraction & -0.0153 & 0.0051 & $<0.005^{\star}$ \\
Creatinine clearance & -0.0104 & 0.0020 & $<0.001^{\star}$ \\
Age & & & 0.34 \\
\hline
\end{tabular}

^Factors in model.

and proatrial natriuretic factor, age, and creatinine clearance as independent variables only proatrial natriuretic factor (1-98) had a significant relation with left ventricular ejection fraction. (coefficient $=-8 \cdot 145$, SE $2 \cdot 834$; $\mathrm{P}=0 \cdot 006)$.

With an ejection fraction of $40 \%$ as the criterion for left ventricular systolic dysfunction, nine patients were classified as having impaired left ventricular function. These nine patients had significantly higher plasma proatrial natriuretic factor concentrations than the remaining 55 patients with a left ventricular ejection fraction $>40 \%$ (1876 (1151) $v$ 1174 (530) $\mathrm{pmol} / \mathrm{l} ; P=0 \cdot 030$ ). Subdividing patients according to the median concentration of circulating proatrial natriuretic factor (1192 pmol/l) yielded a sensitivity of $89 \%$, a specificity of $56 \%$, a positive predictive value of $25 \%$, and a negative predictive value of $97 \%$ for detecting a left ventricular ejection fraction $<40 \%$. Sensitivity, specificity, and predictive values for four arbitrary concentrations of proatrial natriuretic factor (1-98) to detect left ventricular ejection fraction $<40 \%$ are shown in table 2. A logistic regression analysis showed that proatrial natriuretic factor could significantly predict left ventricular ejection fraction below $40 \%$. (Wald test, $\chi^{2}=$ 5.73; $\mathrm{P}<0.02$ ).

With a transmitral E/A ratio of $>0.65$ and $<1.65$ as normal limits ${ }^{18} 44$ patients had normal diastolic function and 20 patients diastolic dysfunction. Patients with abnormal transmitral E/A ratio had significantly higher proatrial natriuretic factor concentrations than patients with a normal E/A ratio (1572 (895) $v 1137$ (523) pmol/l; $P=0.02)$. The median concentration of circulating proatrial natriuretic factor $(1192 \mathrm{pmol} / \mathrm{l})$ had a sensitivity of $75 \%$ and a specificity of $61 \%$ to detect diastolic dysfunction with a positive predictive value of $47 \%$ and a negative predictive value of $84 \%$. Table 2 presents the sensitivity, specificity, and predictive values of four arbitrary concentrations of proatrial natriuretic factor (1-98) in detecting an abnormal E/A ratio.

Of the 64 patients in this study, 39 patients had normal left ventricular function defined as an ejection fraction $>40 \%$ and a normal $\mathrm{E} / \mathrm{A}$ ratio. The remaining 25 patients had an ejection fraction of $<40 \%$ with or without diastolic dysfunction. The median concentration of circulating proatrial natriuretic factor (1192 pmol/l) had a sensitivity of $76 \%$ and a specificity of $67 \%$ in detecting those with abnormal values. This median concentration had a positive predictive value of $59 \%$ and a negative predictive value of $81 \%$ in detecting an ejection fraction $<40 \%$ or an abnormal E/A ratio, or both. The sensitivity, specificity, and predictive values of four arbitrary concentrations of proatrial natriuretic factor (1-98) to detect a left ventricular ejection fraction $<40 \%$ or an abnormal E/A ratio, or both, are also presented in table 2 .

\section{Discussion}

The potential usefulness of atrial peptides as inexpensive and simple tests of cardiac function has recently attracted considerable interest. $^{22} 23$ Proatrial natriuretic factor (1-98) seems to be a promising candidate not only in hospital clinical practice but possibly also in primary health care because of its enhanced stability. This study provides important information on the haemodynamic correlates of proatrial natriuretic factor (1-98) and the clinically relevant confounding factors in assessing this peptide in patients with a recent myocardial infarction.

Atrial natriuretic peptides are released mainly in response to atrial stretch, and their plasma concentrations are dependent on cardiac function and other factors influencing atrial pressures. Left ventricular systolic function is therefore only one of several determinants of proatrial natriuretic factor (1-98) concentrations in plasma. We observed that proatrial natriuretic factor (1-98) was significantly related to left ventricular ejection fraction in a univariate analysis and to renal function and patients' age. In a multivariate model left ventricular ejection fraction and creatinine clearance were independent predictors of circulating proatrial natriuretic factor (1-98) concentration, whereas the association between age and the peptide could be accounted for by the impairment of renal

Table 2 Sensitivity, specificity, and predictive values (percentages) of four arbitrarily selected circulating concentrations of proatrial natriuretic factor (1-98) for detecting left ventricular systolic or diastolic dysfunction, or both, in patients after myocardial infarction

\begin{tabular}{|c|c|c|c|c|c|c|}
\hline & \multicolumn{2}{|c|}{ Ejection fraction $<40 \%$} & \multicolumn{2}{|c|}{ Abnormal E/A ratio } & \multicolumn{2}{|c|}{$\begin{array}{l}\text { Ejection fraction }<40 \% \text { and } \\
\text { abnormal E/A ratio }\end{array}$} \\
\hline & $\begin{array}{l}\text { Sensitivity/ } \\
\text { specificity }\end{array}$ & $\begin{array}{l}\text { Positive predictive } \\
\text { value/negative } \\
\text { predictive value }\end{array}$ & $\begin{array}{l}\text { Sensitivity/ } \\
\text { specificity }\end{array}$ & $\begin{array}{l}\text { Positive predictive } \\
\text { value/negative } \\
\text { predictive value }\end{array}$ & $\begin{array}{l}\text { Sensitivity/ } \\
\text { specificity }\end{array}$ & $\begin{array}{l}\text { Positive predictive } \\
\text { value/negative } \\
\text { predictive value }\end{array}$ \\
\hline \multicolumn{7}{|c|}{ Proatrial natriuretic peptide $(1-98)(\mathrm{pmol} / \mathrm{l})$ : } \\
\hline 500 & $100 / 4$ & $15 / 100$ & $100 / 5$ & $32 / 100$ & $100 / 5$ & $40 / 100$ \\
\hline 1000 & $89 / 42$ & $20 / 96$ & $80 / 46$ & $40 / 83$ & $80 / 49$ & $50 / 80$ \\
\hline 1500 & $56 / 84$ & $36 / 92$ & $40 / 86$ & $57 / 76$ & $40 / 90$ & $71 / 70$ \\
\hline 2000 & $22 / 95$ & $40 / 88$ & $10 / 93$ & $40 / 70$ & $12 / 95$ & $60 / 62$ \\
\hline
\end{tabular}


function with increasing age. In absolute terms plasma concentrations of the peptide seem to be only modestly related to left ventricular ejection fraction and is affected significantly by renal function in this sample of patients with a recent myocardial infarction with or without ventricular dysfunction.

The results of the prevention arm of the studies of left ventricular dysfunction (SOLVD) and the survival and ventricular enlargement (SAVE) trials suggest a benefit of angiotensin converting enzyme inhibition in terms of decreased morbidity or mortality, or both, in patients with asymptomatic left ventricular dysfunction. ${ }^{72}$ As a consequence, there is currently increasing interest in simple, cost effective, and reliable methods for identifying this patient group. ${ }^{22}{ }^{23}$ Although echocardiography and radionuclide ventriculography may provide valuable information in diagnosing both systolic and diastolic ventricular impairment, both methods are time consuming and require expensive equipment and highly skilled staff, and this limits their applicability. After the encouraging report that determination of proatrial natriuretic factor (1-98), in contrast to determination of atrial natriuretic factor (99-126), may identify subjects with asymptomatic left ventricular dysfunction with a sensitivity and specificity of more than $90 \%,{ }^{15}$ measurement of this peptide has emerged as a promising candidate for screening patients with suspected cardiac disease. Moreover, proatrial natriuretic factor (1-98) is an independent predictor of long term prognosis after myocardial infarction, ${ }^{25}$ and its value in predicting mortality one year after myocardial infarction is better than that of atrial natriuretic factor $(99-126) .{ }^{26}$ An additional advantage of measuring proatrial natriuretic factor (1-98) rather than atrial natriuretic factor (99-126) is based on its longer plasma half life and subsequent higher plasma concentration, which permits simplified handling and analysis.

Our results suggest that the association between non-invasive haemodynamic indices and plasma concentrations of proatrial natriuretic factor (1-98) is relatively modest in the acute phase after myocardial infarction. The relation between plasma concentrations of proatrial natriuretic factor (1-98) and left ventricular ejection fraction, although significant, was far weaker than that required if assessment of this peptide should be used as an alternative method to echocardiography in determining low left ventricular ejection fraction in patients with recent myocardial infarction. Results from a limited number of patients indicate that plasma brain natriuretic peptide, a cardiac ventricular hormone, may correlate more strongly with left ventricular ejection fraction after myocardial infarction. ${ }^{27}$ Larger studies are required to determine whether this relation is consistent in an unselected group of patients after infarction. Despite the lack of a close association between plasma proatrial natriuretic factor (1-98) concentrations and left ventricular systolic function, its assay might have some clini- cal application if a normal value indicated a normal left ventricular function-that is, if the test had a high negative predictive value. In fact, this seems to be the case, as shown in table 2 . Hence, a low circulating concentration of proatrial natriuretic factor (1-98) might be used as a screening test to exclude left ventricular dysfunction. If used as a screening test, however, the upper normal limit for circulating proatrial natriuretic factor concentration, as shown by our data, should be adjusted for renal function.

What are the potential reasons for the lack of a close relation between plasma proatrial natriuretic factor (1-98) concentrations and indices of left ventricular function as assessed by echocardiography? Some limitations are inherent in calculating left ventricular ejection fraction by echocardiography. Focal hypokinesia in an area with ischaemic injury may be compensated for by hyperkinesia in an area with normal myocardium. Volumes calculated from cross sectional area tracings are susceptible to errors dependent on the functional status of observed myocardium at the plane of measurement. To minimise these potential errors all volumes were derived from measurements at two orthogonal planes. Probably more important is the fact that the physiological stimulus for atrial natriuretic factor release is atrial stretch. The relation between left ventricular ejection fraction, left ventricular filling pressures, and thus atrial pressures may be compromised by concomitant medical treatment and by variations in the distensibility of the cardiac chamber. Future investigations should therefore aim at determining the sensitivity and specificity of proatrial natriuretic factor values in relation to cardiac filling pressures in patients with cardiac disease.

We found that patients with an abnormal transmitral E/A ratio had significantly higher plasma proatrial natriuretic factor (1-98) concentrations than patients with a normal transmitral E/A ratio. However, we did not try to show a linear relation between these two variables as diastolic dysfunction may result in an abnormally high or abnormally low E/A ratio dependent on the cause of dysfunction. Reduced diastolic distensibility results in a low E/A ratio, whereas a high diastolic filling pressure may result in an abnormally high $\mathrm{E} / \mathrm{A}$ ratio. In this unselected patient population variable systolic function, heart rate, and autonomic tone-all factors known to influence diastolic filling velocities, ${ }^{28-30}$ will obscure a potential association between circulating proatrial natriuretic factor (1-98) concentration and transmitral E/A ratio. Since transmitral E/A ratio seems to reflect left atrial pressure particularly well in instances of concomitant left ventricular systolic dysfunction, ${ }^{31}$ future investigations should probably focus on the potential relation between circulating atrial peptides and diastolic indices in this patient subgroup.

Patients with chronic renal failure, in particular before haemodialysis, have raised plasma concentrations of proatrial natriuretic factor. ${ }^{1732}$ The impact of renal function on 
circulating proatrial natriuretic factor (1-98) in this sample of patients with myocardial infarction without overt renal failure may seem unexpectedly pronounced. The excretion of peptides with a molecular weight of approximately 10000 may, however, be impaired at an earlier stage, with subsequent accumulation of these substances. In contrast to atrial natriuretic factor (99-126), which is rapidly cleared from the circulation by plasma proteases as well as a specific clearance receptor, ${ }^{33}$ proatrial natriuretic factor (1-98) may be predominantly cleared by renal excretion. The relation between plasma concentrations of proatrial natriuretic factor (1-98) and renal function in patients with cardiac disease without overt renal failure is therefore most probably caused by a decreased clearance rate rather than an increased release rate of the peptide into the circulation.

In conclusion, our results suggest that in the early phase after myocardial infarction indices of renal and left ventricular systolic function are independently, albeit modestly, related to plasma concentrations of proatrial natriuretic factor (1-98). Plasma concentrations of proatrial natriuretic factor (1-98) seem to reflect an integral of renal and cardiac performance, rather than specific haemodynamic variables as assessed by non-invasive methods. Although the relation between circulating proatrial natriuretic factor (1-98) and left ventricular ejection fraction is significant, the clinical use of the factor as a surrogate marker of left ventricular dysfunction after myocardial infarction is not recommended. Plasma proatrial natriuretic factor (1-98) concentrations after myocardial infarction may, however, be used clinically to identify patients with a high probability of normal left ventricular systolic and diastolic function.

We express thanks to Rolv Terje Lie, for his expert help with the statistical analysis; Anneli Ambring for measuring Dopple mitral flow velocities; and $R$ Wikh and $T$ Gustavson at the MEDNET laboratory, Gothenburg, Sweden, for expert help in developing the Doppler analysis program. This study was supported by a grant from Merck Research Laboratories (Norwegian and Scandinavian subsidiary).

1 Taylor GJ, O'Neal Humphries J, Mellits ED, Pitt B chulze RA, Grifith LSC, et al. Predictors of clinica course, coronary anatomy and left ventricular function after recovery from acute myocardial infarction. Circulation 1980;62:960-70.

2 Multicenter Post-Infarction Group. Risk stratification and survival after myocardial infarction. $N$ Engl f $\mathrm{Med}$ 1983;309:321-6.

3 White HD, Norris RM, Brown MA, Brandt PWT, Whitlock RM, Wild CJ. Left ventricular end-systolic volume as the major determinant of survival after recovery from myocardial infarction. Circulation 1987;76:44-51.

4 Sharpe N, Smith H, Murphy J, Greaves S, Hart H, Gamble G. Early prevention of left ventricular dysfunction after myocardial infarction with angiotensin-contion after myocardial infarction with angiotensin-co

5 Pfeffer MA, Braunwald E, Moye LA, et al. Effect of captopril on mortality and morbidity in patients with left ventricular dysfunction after myocardial infarction. $N$ Engl $f$ Med 1992;327:669-77.

6 The Acute Infarction Ramipril Efficacy (AIRE) Study Investigators. Effect of ramipril on mortality and morbidity of survivors of acute myocardial infarction with clinical evidence of heart failure. Lancet 1993;342:821-8.

7 SOLVD Investigators. Effect of enalapril on survival in patients with reduced left ventricular ejection fraction and congestive heart failure. $N$ Engl f Med 1991;325 293.

8 Konstam MA, Rousseau MF, Kronenberg MW, Udelson $\mathrm{JE}$, Stewart $\mathrm{D}$, et al. Effects of angiotensin converting enzyme inhibitor enalapril on long-term progression of left ventricular dysfunction in patients with heart failure. Circulation 1992;86:431-8.
9 Konstam MA, Kronenberg MW, Rousseau MF, Udelson $\mathrm{JE}$, et al. Effects of angiotensin converting enzyme inhibitor enalapril on long-term progression of left ventricular dysfunction in patients asymptomatic systolic dysfunction. Circulation 1993;88:2277-83.

10 Swedberg K, Held P, Kjekshus J, Rasmussen K, Ryden L, Wedel $\mathrm{H}$ on behalf of the CONSENSUS II Study Group. Effects of the early administration of enalapril on
mortality in patients with acute myocardial infarction. mortality in patients with acute

11 Bonarjee VVS, Carstensen S, Caidahl K, Nilsen DWT, Edner M, Berning J. Attenuation of left ventricular dilatation after acute myocardial infarction by early initiation of enalapril therapy. Am f Cardiol 1993;72:1004-9.

12 Gruppo Italiano per lo Studio della Sopravvivenza nell'Infarcto Miocardico. GISSI-3: effects of lisinopril and transdermal glyceryi trinitrate singly and together on 6-week mortality and ventricular function after acute myocardial infarction. Lancet 1994;343:1115-22.

13 Ferguson JJ. Meeting highlights. ISIS 4. Circulation 1994; 89:545.

14 Raine AE, Erne P, Burgisser E, et al. Atrial natriuretic peptide and atrial pressure in patients with congestive heart failure. $N$ Engl f Med 1986;315:533-7.

15 Lerman A, Gibbons RJ, Rodeheffer RJ, Bailey KR, McKinley LJ, Heublein DM, et al. Circulating N-terminal atrial natriuretic peptide as a marker for symptomless atrial natriuretic peptide as a marker for symptomles

16 Thibault G, Garcia R, Gutkowska J, Bilodeau J, Lazure C, Seidah NG, et al. The propeptide Asn 1-Tyr 126 is the storage form of rat atrial natriuretic factor. Biochem $\mathcal{F}$ 1987;241:265-72.

17 Sundsfiord JA, Thibault G, Larochelle P, Cantin M. Identification and plasma concentrations of the N-terminal fragment of atrial natriuretic factor in man. $f$ Clin Endocrinol Metab 1988;66:605-10.

18 Spirito P, Maron BJ. Influence of aging on Doppler echocardiographic indices of left ventricular dysfunction. Br Heart f 1988;59:672-9.

19 Klein AL, Cohen GI. Doppler echocardiographic assessment of constructive pericarditis, cardiac amyloidosis, and cardiac tamponade. Clev Clin $f$ Med 1992;59: 278-90.

20 Cockroft DW, Gault MH. Prediction of creatinine clearance from serum creatinine. Nephron 1976;16:31-41.

21 American Society of Echocardiography Commitee on Standards, Subcommitee on Quantitation of TwoStandards, Subcommitee on Quantitation of TwoCrawford $\mathrm{M}$, et al. Recommendations for quantitation of the left ventricle by two-dimensional echocardiography. F Am Soc Echocardiogr 1989;2:358-67.

22 Barnett DB. Diagnosis of symptomless left ventricular dysfunction. Lancet 1993;341:1124-5.

23 Struthers AD. Plasma concentrations of brain natriuretic peptide: will this new test reduce the need for cardiac investigations? Br Heart $\mathcal{f} 1$ 1993;70:397-8.

24 SOLVD Investigators. Effect of enalapril on mortality and the development of heart failure in asymptomatic patients with reduced left ventricular ejection fractions. N Engl F Med 1992;327:685-91.

25 Hall C, Rouleau J, Moyé L, et al. N-terminal proatrial natriuretic factor, an independent predictor of long-term prognosis after myocardial infarction. Circulation 1994; 89:1934-42.

26 Omland T, Bonarjee VVS, Nilsen DWT, Sundsfjord JA, Lie RT, Thibault G, et al. Prognostic significance of Nterminal pro-atrial natriuretic factor (1-98) in acute myocardial infarction: comparison with atrial natriuretic factor $(99-126)$ and clinical evaluation. Br Heart $\mathcal{f} 1993$; factor $(99-126$.

27 Motwani JG, MacAlpine H, Kennedy N, Struthers AD. Plasma brain natriuretic peptide as an indicator for angiotensin-converting-enzyme inhibition after myocardial infarction. Lancet 1993;341:1109-13.

28 Danielsen $\mathrm{R}$, Nordrehaug JE, Vik-Mo $\mathrm{H}$. Importance of adjusting left ventricular diastolic peak filling rate for heart rate. Am $\mathcal{F}$ Cardiol 1988;61:489-91.

29 Himura Y, Kumada T, Kambayashi M, Hayashida W, Ishikawa N, Nakamura Y, et al. Importance of left ventricular systolic function in the assessment of left ventriculcr diastolic function with Doppler transmitral flow ular diastolic function with Doppler transmitral flow

30 Johannessen KA, Cerqueira M, Veith RC, Stratton JR. Influence of sympathetic stimulation and parasympaInfluence of sympathetic stimulation and parasympathetic withdrawal on Doppler echocardiographic left ventricular diastolic filling velocities

31 Pozzoli M, Capomolla S, Opasich C, Reggiani R, Calsamiglia G, Cobelli $F$, et al. Left ventricular filling pattern and pulmonary wedge pressure are closely related in patients with recent anterior myocardial infarction and left ventricular dysfunction. Eur Heart $\mathcal{f}$ 1992;13:1067-73.

32 Buckley MG, Sagnella GA, Markandu ND, Singer DRJ, MacGregor GA. Immunoreactive N-terminal pro-atrial natriuretic peptide in human plasma: plasma levels and comparisons with a-human atrial natriuretic peptide in essential hypertension cardiac transplant and chronic renal failure. Clin Sci (Colch) 1989;77:573-9.

33 Almeida FA, Suzuzki RM, Scarborough JA, et al. Clearance function of type C-receptors of atria natriuretic factor in rats. $A m \mathcal{F}$ Physiol 1989;256: R469-75. 